\title{
O envolvimento da Ciência Brasileira no diagnóstico da COVID-19
}

\section{The involvement of Brazilian Science in the diagnosis of COVID-19}

\author{
Laís Moreira Nogueira ${ }^{1}$ \\ Michelli dos Santos ${ }^{1}$ \\ Amanda Aparecida Batista ${ }^{1}$ \\ Ana Clara Gontijo Maia ${ }^{1}$ \\ Sílvio Elisei Carvalho Júnior ${ }^{1}$ \\ André Vinícius Fernandes Ferreira ${ }^{1}$ \\ Jonatas Oliveira Silva ${ }^{1}$ \\ Rodolfo Cordeiro Giunchetti ${ }^{2}$ \\ Mariana Campos da Paz ${ }^{1}$ \\ Alexsandro Sobreira Galdino ${ }^{1}$ \\ Juliana Machado ${ }^{1}$
}

\section{RESUMO}

A COVID-19 é uma doença infecciosa de fácil disseminação que rapidamente se tornou uma pandemia. Um dos desafios enfrentados é o diagnóstico, visto que os métodos utilizados possuem limitações, como a dependência de insumos importados. 0 objetivo desse estudo foi divulgar ações e estratégias de universidades e institutos de pesquisa para o diagnóstico da COVID-19 no Brasil. Deste modo, foi realizada uma busca de patentes no Instituto Nacional da Propriedade Industrial (INPI) e em sites de notícias oficiais no período de 25 de fevereiro a 10 de maio de 2020. Além disso, uma análise comparativa das iniciativas foi realizada. Neste estudo não foram encontradas patentes relacionadas ao tema, porém 88 iniciativas abordando diagnósticos molecular e imunológico, além de métodos alternativos foram selecionadas. Por meio da análise das iniciativas, foi possível demonstrar a relevância e aplicabilidade destas ações dentro do contexto da pandemia, visto que o diagnóstico se tornou uma estratégia fundamental para o controle da COVID-19.

\section{PALAVRAS-CHAVE}

Coronavírus; Pandemia; Universidades; Institutos de pesquisa; Diagnóstico.

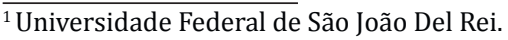

${ }^{2}$ Laboratório de Biologia das Interações Celulares, Universidade Federal de Minas Gerais, Belo Horizonte.
} 


\section{ABSTRACT}

COVID-19 is an easily spreadable infectious disease that quickly became a pandemic. One of the challenges faced is the diagnosis, since the methods used have limitations, such as dependence on imported inputs. The objective of this study was to publicize actions and strategies of universities and research institutes for the diagnosis of COVID-19 in Brazil. Thus, a search for patents at the National Institute of Industrial Property (INPI) and on official news sites was carried out from February 25 to May 10, 2020. In addition, a comparative analysis of the initiatives was carried out. In this study, no patents related to the topic were found, however 88 initiatives addressing molecular and immunological diagnoses, in addition to alternative methods were selected. Through the analysis of the initiatives, it was

possible to demonstrate the relevance and applicability of these actions within the context of the pandemic, since the diagnosis has become a fundamental strategy for the control of COVID-19.

\section{KEYWORDS}

Coronavírus; Pandemic; Universities; Research institutes; Diagnosis. 


\section{INTRODUÇÃO}

Em dezembro de 2019, uma epidemia viral de etiologia desconhecida foi relatada em Wuhan, província de Hubei, na China. Posteriormente, a doença foi identificada como Corona Virus Disease - 19 (COVID-19), causada por um tipo de Coronavírus ( $\mathrm{CoV}$ ) altamente contagioso, capaz de causar sintomas como pneumonia, febre, dificuldade respiratória e infecção pulmonar (ROTHAN; SIDDAPPA, 2020).

Os CoV pertencem à família Coronaviridae e causam infecções enzoóticas em aves e mamíferos e, nas últimas décadas, demonstraram a capacidade de infectar humanos. Os surtos da síndrome respiratória aguda grave (SARS) em 2002 e da síndrome respiratória do Oriente Médio (MERS), com taxas de mortalidade de 10\% e $35 \%$, respectivamente, demonstraram que os $\mathrm{CoV}$ tem o potencial de desencadear pandemias (CUI; LI; SHI, 2019; TORTORICI; VEESLER, 2019).

O CoV responsável pela COVID-19 foi nomeado oficialmente coronavírus 2 causador da síndrome respiratória aguda grave (SARS-CoV-2) e, rapidamente se espalhou por diversos países. Por provocar inúmeras mortes pelo mundo, foi declarada uma Emergência de Saúde Pública de Interesse Internacional pela Organização Mundial de Saúde (OMS) (ADHIKARI et al., 2020).

Atualmente, o diagnóstico da COVID-19 é realizado por meio de métodos moleculares, como a transcrição reversa do RNA viral seguida de reação em cadeia da polimerase (RT-PCR). Contudo, é uma técnica que exige equipamentos sofisticados e profissionais treinados para a sua realização (GUO et al., 2020). Para complementar os métodos moleculares, testes sorológicos estão sendo realizados para auxiliar no diagnóstico da COVID-19. Além de atuarem como indicadores de infecção podem fornecer informações sobre o tempo de exposição ao vírus (UDUGAMA et al., 2020).

Diante do crescente número de infectados, da ausência de vacinas e tratamentos específicos cientificamente comprovados, o desenvolvimento de um método de detecção com elevada sensibilidade e especificidade é de extrema importância para a adoção de medidas de mitigação do impacto da pandemia (ADHIKARI et al., 2020).

Assim, este trabalho aborda as contribuições das universidades públicas e institutos de pesquisa do Brasil no auxílio do enfrentamento da pandemia, especial- mente aquelas relacionadas ao desenvolvimento de novos métodos de diagnóstico da COVID-19. Atualmente, várias pesquisas com a finalidade de se desenvolver métodos de diagnóstico específicos, rápidos e acessíveis encontram-se em andamento. As tecnologias vindouras serão de grande valia para diminuir a subnotificação da doença, além de contribuir na identificação dos indivíduos possivelmente imunes. Dessa forma, essas ações podem auxiliar na tomada de decisões referentes à quarentena e à flexibilização responsável do retorno às atividades regulares.

\section{METODOLOGIA}

\section{Busca de patentes relacionadas ao diagnóstico da COVID-19}

Com a finalidade de se analisar as iniciativas de universidades e institutos de pesquisa brasileiros no âmbito da propriedade industrial, foi realizada uma busca no banco de dados do Instituto Nacional da Propriedade Industrial (INPI) por patentes relacionadas ao diagnóstico da COVID-19. A busca por patentes que contivessem as palavras-chave: COVID-19, novo coronavírus, SARS-CoV-2, diagnóstico COVID-19, diagnóstico novo coronavírus, diagnóstico SARS-CoV-2 e diagnóstico coronavírus, no título ou resumo, foi feita no período entre 25 de fevereiro a 10 de maio de 2020.

\section{Busca de iniciativas de universidades e institutos de pesquisa brasileiros para o diagnóstico da COVID-19}

Foi feita uma busca em sites oficiais de universidades, institutos de pesquisa e agências de fomento por notícias abordando as estratégias realizadas para o diagnóstico da COVID-19. A pesquisa foi realizada no período entre 25 de fevereiro a 10 de maio de 2020 e abrangeu iniciativas de pesquisa e extensão. As palavras-chave utilizadas foram: COVID-19, novo coronavírus, SARS-CoV-2, pandemia.

\section{Critérios de elegibilidade}

A elegibilidade das notícias e reportagens encontradas teve como critério de inclusão qualquer reportagem 
que abordasse pesquisas e iniciativas relacionadas ao diagnóstico da COVID-19. Os critérios de exclusão abrangeram notícias sobre treinamentos de profissionais, iniciativas de startups e pesquisas sobre tratamentos e/ou vacinas.

\section{Análise comparativa dos métodos de diagnóstico}

Após a seleção das iniciativas foi realizada uma análise comparativa das ações com relação aos parâmetros: (i) tempo de detecção de vírus/anticorpos; (ii) tempo entre execução do exame e liberação do resultado; (iii) custo estimado; (iv) possibilidade de aplicação em larga escala. Para cada um destes parâmetros foi atribuída uma nota de 1 a 5, fundamentada na notícia de cada iniciativa disponível eletronicamente e na literatura científica. Os critérios estabelecidos para a atribuição das notas são mostrados na Tabela 1 . Vale ressaltar que a análise não foi aplicada para as iniciativas que envolvem diagnóstico por imagem, inteligência artificial e softwares pois não foi possível mensurar os parâmetros para estas ações.

Tabela 1. Critérios estabelecidos para atribuição da nota.

\begin{tabular}{llllll}
\hline Parâmetros & $\mathbf{1}$ & $\mathbf{2}$ & $\mathbf{3}$ & $\mathbf{4}$ & $\mathbf{5}$ \\
\hline $\begin{array}{l}\text { Detecção do mate- } \\
\text { rial genético/ }\end{array}$ & $\begin{array}{l}\text { Detecção entre } \\
\text { a quarta e quin- } \\
\text { ta semana após } \\
\text { anticono/ }\end{array}$ & $\begin{array}{l}\text { Detecção entre } \\
\text { a terceira e } \\
\text { quarta semana } \\
\text { após sintomas }\end{array}$ & $\begin{array}{l}\text { Detecção entre } \\
\text { a segunda e } \\
\text { terceira sema- } \\
\text { na após sinto- } \\
\text { mas }\end{array}$ & $\begin{array}{l}\text { Deteção en- } \\
\text { tre a primeira } \\
\text { e segunda se- } \\
\text { mana após } \\
\text { sintomas }\end{array}$ & $\begin{array}{l}\text { Detecção na } \\
\text { primeira se- } \\
\text { mana após } \\
\text { sintomas }\end{array}$ \\
$\begin{array}{l}\text { Tempo entre execu- } \\
\text { ção do exame e libe- } \\
\text { ração do resultado }\end{array}$ & $\begin{array}{l}\text { Mais de 24 ho- } \\
\text { ras }\end{array}$ & $\begin{array}{l}1,1 \text { horas a 5 } \\
\text { horas }\end{array}$ & $\begin{array}{l}31 \text { minutos a } 1 \\
\text { hora }\end{array}$ & $\begin{array}{l}1,1 \text { minutos a } \\
30 \text { minutos }\end{array}$ & $\begin{array}{l}1 \text { segundo a } \\
60 \text { segundos }\end{array}$ \\
$\begin{array}{l}\text { Custo } \\
\text { estimado }\end{array}$ & $\begin{array}{l}\text { Mais de 100 re- } \\
\text { ais }\end{array}$ & 51 a 100 reais & 31 a 50 reais & 11 a 30 reais & 1 a 10 reais \\
$\begin{array}{l}\text { Possibilidade de } \\
\text { aplicação em larga } \\
\text { escala }\end{array}$ & $20 \%$ & & $60 \%$ & & \\
\end{tabular}

Os scores de 1 a 5 foram atribuídos e ilustram os diferentes parâmetros considerados: (i) Detecção do material genético/antígeno/ anticorpo; (ii) Tempo entre execução do exame e liberação do resultado; (iii) Custo estimado; (iv) Possibilidade de aplicação em larga escala. Deste modo, cada teste avaliado neste estudo foi categorizado de acordo com o score atribuído ao parâmetro que o caracteriza.

\section{RESULTADOS E DISCUSSÃO}

\section{Busca de patentes relacionadas ao diagnóstico da COVID-19}

Em cenário de pandemia, como enfrentado atualmente, a ciência e tecnologia vêm como grandes aliadas no desenvolvimento de vacinas, medicamentos, métodos diagnósticos, equipamentos hospitalares e de proteção individual. Neste âmbito, a proteção dos direitos relativos à propriedade industrial ganha destaque como ferramenta necessária para promover uma melhoria na estrutura econômica do país, incentivando a criação de novas tecnologias e propagando conhecimento (ZUCOLOTO; MIRANDA; PORTO, 2020).

$\mathrm{Na}$ busca realizada no banco de patentes do INPI, nenhuma patente relacionada diretamente ao diagnóstico da COVID-19 foi encontrada durante o período estipulado. Esse resultado não reflete a ausência de patentes depositadas no Brasil. Embora o INPI tenha criado uma modalidade de trâmite prioritário de pedidos de patente relacionados a produtos e processos farmacêuticos e a equipamentos e/ou materiais de uso em saúde, para o diagnóstico, profilaxia e tratamento da COVID-19 (INPI, 2020), uma possível explicação seria a demora no exame dos pedidos de patente devido à grande demanda. 
Recentemente o INPI divulgou um estudo sobre o "Panorama das patentes depositadas no INPI descrevendo métodos de diagnóstico para coronavírus e outras viroses respiratórias" que teve como objetivo divulgar as tecnologias que podem contribuir no enfrentamento da pandemia. No estudo, foram encontrados 141 pedidos de patente depositados no INPI que se referem ao diagnóstico de viroses respiratórias. Destes, 65 pedidos foram relacionados aos $\mathrm{CoV}$ como potencial alvo de testes de diagnóstico. As patentes foram depositadas principalmente entre os anos de 2004-2012, período em que ocorreram as epidemias de SARS e MERS, respectivamente. Contudo, os principais depositantes foram empresas e instituições de pesquisas internacionais (WEID, 2020).

Estudos como este realizado pelo INPI auxiliam no enfrentamento da COVID-19 uma vez que muitos desses produtos e processos patenteados podem virar produtos que irão auxiliar em métodos de diagnósticos mais sensíveis e específicos.

\section{Busca de iniciativas de universidades e institutos de pesquisa brasileiros para o diagnóstico da COVID-19}

Com o uso das palavras-chave escolhidas, foram identificadas 150 notícias referentes às iniciativas de universidades e institutos de pesquisa brasileiros para o diagnóstico da COVID-19. Porém, após o processo de triagem de acordo com os critérios de elegibilidade descritos no item Metodologia, 88 notícias foram selecionadas para análise. 0 fluxograma da pesquisa é mostrado na Figura 1.

\section{Contribuições das universidades públicas para o desenvolvimento de novos métodos de diagnóstico da COVID-19}

Com o intuito de ampliar a capacidade diagnóstica da COVID-19 e desenvolver novos testes, pesquisadores de universidades de várias regiões do país estão traba-

Figura 1. Fluxograma de busca e seleção das estratégias realizadas pelas universidades e institutos de pesquisa.

Registros excluídos Não atenderam aos critérios de elegibilidade estabelecidos $\mathrm{n}=62$ 
lhando no processo de elaboração de métodos que consigam suprir a grande demanda diante da atual pandemia. As abordagens adotadas pelos diversos grupos de pesquisa serão discutidas a seguir.

\section{Diagnósticos Moleculares}

Em fevereiro de 2020, a Universidade Federal da Bahia (UFBA) anunciou a execução do exame de detecção do SARS-CoV-2 em apenas 3 horas pelo método de RT-PCR, reduzindo em 45 horas a espera pelo resultado. 0 exame é feito através do material genético extraído de secreções respiratórias recolhidas de pacientes suspeitos (PRADO, 2020).

Diante do crescente número de casos da doença e da necessidade de desenvolvimento de novos métodos de diagnóstico, no mês de abril, pesquisadores do Instituto de Ciências Biomédicas da Universidade de São Paulo (USP) desenvolveram um teste molecular utilizando o método de PCR convencional. Os testes são realizados com reagentes nacionais e poderão fornecer resultados em 4 horas garantindo a mesma sensibilidade e especificidade dos diagnósticos feitos com a RT-PCR (BERNARDES, 2020a).

No intuito de agilizar o diagnóstico local e contribuir para o controle da doença, uma equipe do Laboratório de Estudos de Vírus Emergentes do Instituto de Biologia da Universidade Estadual de Campinas (Unicamp), em colaboração com a Faculdade de Ciências Médicas e o Laboratório Nacional de Biociências estão atuando no processo de elaboração de um teste molecular, por meio da RT-PCR, para a detecção da COVID-19. 0 estudo utiliza protocolo desenvolvido por pesquisadores do German Center for Infection Research e contou com a colaboração de outras instituições, como o Centro Nacional de Pesquisa em Energia e Materiais e USP (COLL, 2020).

Além das iniciativas mencionadas, uma metodologia inovadora foi proposta por pesquisadores do Instituto de Física de São Carlos da USP em parceria com o Hospital de Amor de Barretos. A iniciativa consiste em adaptar o método que utiliza genossensores, que detectam o material genético de tumores cancerígenos dentro de poucos minutos, ao diagnóstico da COVID-19. 0 dispositivo utilizado é simples e portátil, permitindo que o exame seja feito em qualquer local e não apenas em laboratórios (BERNARDES, 2020b).

\section{Diagnósticos Imunológicos}

Na Universidade Federal do Rio de Janeiro (UFRJ), pesquisadores do Laboratório de Engenharia de Cultivos Celulares e do Laboratório de Virologia Molecular estão atuando no desenvolvimento de um teste sorológico a partir das proteínas do SARS-CoV-2. 0 teste rápido se baseia na detecção de anticorpos permitindo diagnosticar se a pessoa é positiva para COVID-19 e revelar aquelas que já foram infectadas e não apresentaram sintomas (UFRJ, 2020)

Já o Laboratório de Virologia do Departamento de Microbiologia do Instituto de Ciências Biomédicas (ICB) em conjunto com o Laboratório do Instituto de Química da USP estão trabalhando na elaboração de um teste rápido, porém, por meio da detecção do vírus em amostras de secreções da garganta. 0 desenvolvimento do teste envolve a produção de fragmentos de proteínas de superfície do vírus para obtenção de anticorpos que serão utilizados na montagem do teste (BERNARDES, 2020a).

Além disso, membros do ICB da USP estão desenvolvendo um teste sorológico baseado no ensaio de imunoabsorção enzimática (ELISA). Este tipo de teste que identifica os anticorpos IgM/IgG no sangue ou plasma sanguíneo já está disponível no mercado e os equipamentos necessários para realização deste teste estão disponíveis na maioria dos laboratórios de análises clínicas do país (BERNARDES, 2020a)

Pode-se destacar ainda os pesquisadores da Universidade Federal de Pelotas (UFPel), que estão atuando na criação de testes sorológicos nacionais, onde antígenos selecionados a partir de ferramentas de imunoinformática serão produzidos por meio da engenharia genética. Dos antígenos triados, dez já foram selecionados para a produção de genes sintéticos em bactérias geneticamente modificadas e serão utilizados no desenvolvimento do teste (UFPEL, 2020).

Neste mesmo segmento, uma equipe do Programa de Pós Graduação (PPG) em Genética da Universidade Federal de Pernambuco (UFPE) propõe a utilização da levedura Pichia Pastoris como biofábrica para a produção de proteínas visando o desenvolvimento de um diagnóstico sorológico. Além disso, pesquisadores do PPG em Nutrição, Atividade Física e Plasticidade Fenotípica propuseram a realização do diagnóstico sorológico semiquantitativo a partir da pesquisa do IgA em soro 
de pacientes na fase aguda da doença. De maneira complementar, pesquisadores do PPG em Inovação Terapêutica estão desenvolvendo testes rápidos do tipo imunocromatográfico de fluxo lateral e biodispositivos sensores flexíveis descartáveis (UFPE, 2020).

Somado a isso, pesquisadores da Universidade Estadual Paulista (Unesp) estão engajados no desenvolvimento de um teste rápido. 0 método consiste em uma tira de papel cromatográfico com nanopartículas de ouro para identificação, em 15 minutos, de anticorpos IgG e IgM contra o SARS-CoV-2 presentes na saliva ou outra secreção do paciente (ZIEGLER, 2020).

Além de todas as propostas mencionadas, pesquisadores da Universidade Federal de São João Del Rei (UFSJ) e da Universidade Federal de Minas Gerais (UFMG) juntamente com as startups Chimera Diagnósticos - Saúde Humana e Animal e VetSolutions também trabalham no desenvolvimento de testes rápidos nacionais. 0 impacto da iniciativa se deve ao fato de que com a aplicação da tecnologia nacional, o valor por unidade pode cair de aproximadamente $\mathrm{R} \$ 300,00$ (valor atual dos testes importados) para $\mathrm{R} \$ 5$, representando uma redução de cerca de $98 \%$ no valor. A parceria UFSJ/ UFMG visa obter testes com grande sensibilidade/especificidade para disponibilizar a tecnologia para o Sistema Único de Saúde (SUS) (ASCOM, 2020).

\section{Diagnóstico por imagem}

Além dos diagnósticos molecular e imunológico, o diagnóstico por imagem é uma alternativa para a detecção de casos suspeitos da COVID-19. Com base nesta premissa, pesquisadores de diferentes instituições propuseram iniciativas para auxiliar no enfrentamento da pandemia.

Pesquisadores do Laboratório de Imagem, Sinais e Acústica e do Laboratório de Tecnologia da Tomada de Decisão da Universidade de Brasília (UnB) desenvolveram um sistema que consegue determinar, em segundos, casos suspeitos da COVID-19 por meio da leitura de radiografias. Neste método, profissionais de saúde irão carregar a imagem em um sistema online que irá efetuar a análise por meio de algoritmos, fornecendo a identificação de casos suspeitos (GOMES, 2020).

Neste mesmo segmento, um projeto da Universidade Estadual de Maringá (UEM), tem como objetivo iden- tificar a pneumonia causada pela COVID-19 utilizando imagens de radiografias de tórax. Denominada RYDLS-20, a base de dados desenvolvida está disponível publicamente para que outros cientistas também possam utilizá-la (PUPIM, 2020).

De maneira semelhante, pesquisadores do Laboratório de Computação de Sistemas Inteligentes, juntamente com integrantes do Departamento de Informática da Universidade Federal do Paraná (UFPR) buscaram desenvolver um projeto para melhorar a identificação de padrões causados pela COVID-19 através de imagens de raio-x. 0 método constitui uma alternativa promissora para incorporação em equipamentos médicos e até mesmo em telefones celulares, como auxílio na triagem diagnóstica de casos suspeitos (SANTOS, 2020; CUBAS, 2020).

Pesquisadores da Faculdade de Ciências Médicas da Universidade Estadual do Rio de Janeiro (UERJ) também atuam nesse segmento. A iniciativa propõe a utilização da Ultrassonografia de Tórax como alternativa para a avaliação dos casos graves que necessitam de acompanhamento mais efetivo, por ser um exame mais rápido e barato, além de diminuir a exposição do paciente à radiação (UERJ, 2020).

Adicionalmente, o desenvolvimento de um Sistema chamado IKONOS é uma iniciativa do PPG em Engenharia Biomédica da UFPE. Trata-se de um sistema inteligente cujo objetivo é otimizar o diagnóstico através de radiografias do tórax (UFPE, 2020). Da mesma forma, pesquisadores da Universidade Federal de Santa Catarina (UFSC) propõem a classificação de imagens de raio-X e tomografias da região torácica de pacientes suspeitos por meio da inteligência artificial (DALLANHOL, 2020).

Além disso, pesquisadores do Departamento de Ciência da Computação da Universidade Federal Rural do Rio de Janeiro (UFRRJ) desenvolveram um aplicativo chamado XRayCovid-19 para avaliar se a imagem de radiografia possui o padrão associado à COVID-19 ou outras doenças respiratórias (UFRRJ, 2020).

Somado a todos esses esforços, pesquisadores da USP juntamente com o Hospital das Clínicas desenvolveram a RadVid19. Esta plataforma, reúne dados sobre casos do Brasil e tem como referência imagens de radiografias e tomografias da região do tórax. A RadVid19 possui um algoritmo que avaliará as imagens em poucos segundos e fornecerá informações de diferentes está- 
gios da evolução da doença para que associações entre quadros radiológicos e condições clínicas e laboratoriais sejam realizadas (USP, 2020).

\section{Inteligência artificial não associada a exames de imagem}

O Hospital Universitário do Oeste do Paraná iniciou um estudo para a detecção da COVID-19, por meio de exames de rotina como o hemograma. 0 algoritmo do estudo reúne, padrões para identificar se o paciente tem pré-disposição positiva ou negativa para a doença. 0 projeto conta com o auxílio do curso de Ciência da Computação, da Universidade Estadual do Oeste do Paraná (Unioeste) (UNIOESTE, 2020).

Além disso, pesquisadores do Programa de Pós Graduação em Engenharia Biomédica da UFPE propõem o desenvolvimento do Sistema Heg.IA que consiste em um protótipo de um sistema de apoio ao diagnóstico com base em exames de sangue (UFPE, 2020).

Ainda no âmbito da inteligência artificial, pesquisadores do laboratório Minds (Machine Intelligence and Data Science) da Escola de Engenharia da UFMG juntamente com a Faculdade de Medicina, do Hospital das Clínicas e do Departamento de Computação da Universidade Federal de Ouro Preto (UFOP) propõem a criação de um software baseado em inteligência artificial, visando ajudar na detecção automática de síndromes respiratórias agudas. $\mathrm{O}$ funcionamento do software se dá por meio da análise de propriedades sanguíneas e de imagens de exames clínicos de rotina, garantindo mais precisão nas avaliações e maior agilidade e praticidade na tomada de decisões pelo profissional de saúde (ESPINDOLA, 2020).

Pesquisadores da Unicamp e USP, também trabalham no desenvolvimento de um teste rápido para COVID-19 capaz de identificar a infecção em poucos minutos. 0 teste é baseado em uma espécie de "impressão digital" do SARS-CoV-2, onde biomarcadores detectam a presença tanto da COVID-19 quanto de outras doenças relacionadas (ARRUDAS, 2020).

\section{Métodos Espectroscópicos}

Pesquisadores do Instituto de Física da Universidade Federal de Mato Grosso do Sul (UFMS), em parceria com a Fundação Oswaldo Cruz (Fiocruz/MS), estão em busca do desenvolvimento de um método rápido e acessível baseado na espectroscopia óptica em amostras de biofluidos provenientes de pacientes. As maiores vantagens da técnica são: a liberação dos resultados em cerca de cinco minutos e o baixo custo, visto que requer apenas um espectrômetro e dispensa o uso de reagentes (AMIN, 2020).

Além disso, pesquisadores do Instituto de Ciências Biomédicas da Universidade Federal de Uberlândia (ICBIM/UFU), em parceria com a Universidade de Saskatchewan estudam a possibilidade de diagnóstico da COVID-19 pela saliva utilizando a Espectroscopia ATR-FTIR. A intenção é o desenvolvimento de um método não invasivo e que poderá ser coletado pelo próprio paciente (BORGES, 2020).

\section{Contribuições dos institutos de pesquisa brasileiros para o avanço do diagnóstico da COVID-19}

Além das iniciativas das universidades públicas brasileiras, pesquisadores de Institutos também estão contribuindo de maneira efetiva no enfrentamento da pandemia.

\section{Diagnósticos Moleculares}

Desde abril do ano de 2020 a FIOCRUZ vem se dedicando ao desenvolvimento de kits para o diagnóstico do SARS-CoV-2. Estes kits desenvolvidos pelos institutos de Tecnologia em Imunobiológicos e de Biologia Molecular do Paraná utilizam a técnica RT-PCR e têm como objetivo identificar um fragmento específico do genoma do vírus (FIOCRUZ, 2020a).

Além da FIOCRUZ, o Instituto de Pesquisas Tecnológicas do Estado de São Paulo (IPT), juntamente com a Empresa Brasileira de Pesquisa e Inovação Industrial (Embrapii) e a startup Aptah BioSciences busca o desenvolvimento de um teste rápido. Esse teste possui como diferencial a identificação da presença de material genético do SARS-CoV-2 na saliva do paciente e tem como expectativa uma leitura rápida que possa ajudar na triagem do atendimento (IPT, 2020).

\section{Diagnósticos Imunológicos}

Pesquisadores do Centro de Biotecnologia da Amazônia (CBA) estão se empenhando no desenvolvimento 
de kits de diagnóstico rápidos para a COVID-19. 0 resultado poderá ser obtido em minutos utilizando-se gotas de sangue ou saliva. 0 objetivo final é que o centro consiga, além da produção dos kits, o fornecimento de anticorpos e antígenos suficientes para empresas brasileiras (RIOS, 2020).(RIOS, 2020).

Utilizando uma abordagem diferente, pesquisadores do Centro de Desenvolvimento de Materiais Funcionais (CDMF) e do Centro de Pesquisa, Inovação e Difusão (CEPID), com o apoio da FAPESP, estão desenvolvendo um sensor eletroquímico que utiliza nanopartículas de óxido de zinco para detectar a proteína do SARS-CoV-2 através da secreção do nariz ou garganta de pacientes com suspeita da infecção (ZIEGLER, 2020). A equipe está buscando parcerias com grupos da UNICAMP para modificar a base sensora com padrões do tamanho e forma do vírus, realizando assim a sua detecção diretamente e produzindo um teste rápido 100\% nacional e com custo reduzido. 0 resultado desse teste é fornecido em 15 minutos em forma de gráficos e pode ser obtido pelo uso de um equipamento portátil e exibido em aplicativos de smartphones (ZIEGLER, 2020).

\section{Métodos utilizando sensores}

O Instituto Nacional de Ciência e Tecnologia em Teranóstica e Nanobiotecnologia (INCT TeraNano), localizado na Universidade Federal de Uberlândia (UFU), em parceria com a empresa Imunoscan, está trabalhando no desenvolvimento de dois tipos de sensores para o diagnóstico da COVID-19. 0 primeiro é baseado em um sensor fotônico, uma tecnologia a laser capaz de fragmentar a saliva possibilitando o diagnóstico da COVID-19. Trata-se de um equipamento importado, porém vantajoso por dispensar o uso de reagentes. Ademais, o método apresenta grande agilidade, permitindo que o resultado fique pronto em menos de um minuto, além de possuir capacidade de processar entre 400 e 500 resultados por dia. O segundo método em desenvolvimento é um sensor eletroquímico portátil, semelhante a um pen drive, que possui um microchip onde serão depositadas gotas de saliva. 0 sensor poderá ser conectado a um smartphone e permitirá a detecção tanto do vírus através da saliva, quanto de anticorpos através do sangue. 0 diagnóstico poderá ficar pronto em cerca de um minuto, permitindo uma rápida triagem da doença. Será usado um microchip por paciente e o sensor será destinado aos profissionais de saúde (FAPEG, 2020).

\section{Análise comparativa das iniciativas levantadas}

Como já foi demonstrado, as universidades e os institutos de pesquisa brasileiros vêm contribuindo de forma significativa para auxiliar na demanda de desenvolvimento de novos testes. Nesse sentido, foi realizada uma análise comparativa das iniciativas levantadas relacionadas a métodos de diagnóstico, de forma a demonstrar a relevância e a aplicabilidade destas ações dentro do contexto da pandemia, como é mostrado no Quadro 1.

Como pode ser visto no Quadro 1, os testes propostos empregam diferentes abordagens que permitem que eles sejam utilizados para complementar os testes atualmente disponíveis. Os diagnósticos clínico, laboratorial e por imagem são de grande importância para auxiliar profissionais de saúde na identificação de casos suspeitos (RODRIGUEZ-MORALES et al., 2020).

O teste laboratorial padrão para a detecção da COVID-19 em todo o mundo continua sendo a RT-PCR. Apesar da sensibilidade analítica do método ser comumente elevada, a realização dos testes moleculares exige infraestrutura complexa com adoção de medidas de biossegurança adequadas e profissionais capacitados. Somado a isso, o alto custo do teste limita a sua aplicação em larga escala (ZAINOL et al., 2020; LEE et al., 2020).

Nesse contexto, os testes imunológicos podem ser empregados para contornar estas limitações. Quando comparados aos métodos moleculares, apresentam uma execução mais simples além de exigirem menos infraestrutura e apresentarem custo inferior. Os testes baseados na detecção de anticorpos permitem a avaliação de infecções assintomáticas, subclínicas e sintomáticas. Por meio de dados soroepidemiológicos é possível realizar uma estimativa da proporção de indivíduos infectados e da epidemiologia da doença (ZAINOL et al., 2020).

Resultados encontrados por Long e colaboradores (2020) demonstram a relevância de testes para detecção de anticorpos específicos para SARS-CoV-2 para complementar o diagnóstico molecular de casos suspeitos, com resultados negativos para RT-PCR. Além disso, podem ser utilizados para a investigação de infecções 
assintomáticas em contatos próximos a pacientes com a doença. Tais resultados reforçam a importância do desenvolvimento de novos testes imunológicos para uma avaliação mais precisa da abrangência da COVID-19 (LONG et al., 2020).

O Quadro 1 mostra que os métodos de diagnóstico propostos apresentam suas particularidades. Alguns são mais apropriados para o diagnóstico na fase aguda pois, permitem a detecção do material genético ou de proteínas do vírus. Por outro lado, embora os métodos para detecção de anticorpos não sejam adequados para a fase aguda, eles exercem um papel importante no diagnóstico de indivíduos assintomáticos e na investigação soroepidemiológica da COVID-19.

Além disso, foi verificado que métodos alternativos que empregam tecnologias como sensores e espectroscopia vêm como ferramentas relevantes para complementar os métodos tradicionais. Grande parte destas abordagens se destaca por proporcionar um resultado rápido e apresentar um baixo custo.

Por fim, a possibilidade de aplicação em larga escala e o baixo custo de alguns testes propostos representam a esperança de testagem em massa como forma de achatar a curva de disseminação do vírus no país.

\section{Outras ações para o enfrentamento da pandemia}

Diante da destacada capacidade técnico-científica e da disponibilidade de equipamentos para a realização de testes de diagnóstico da COVID-19, diversas instituições públicas de ensino e pesquisa se mobilizaram e desenvolveram outras estratégias para auxiliar na demanda da saúde pública. Dessa maneira, criaram plataformas e unidades de apoio para ampliar processamento de amostras, cederam seus equipamentos para que instituições estaduais aumentassem sua capacidade de detecção, laboratórios foram reorganizados e reade- quados para atender ao fluxo dos testes e aos procedimentos de biossegurança recomendados pela OMS. Além disso, para conter o avanço da pandemia e contribuir para estudos epidemiológicos, se mobilizaram a realizar testes rápidos em visitas domiciliares assim como diagnóstico molecular a partir de amostras coletas em sistema Drive-Thru. As instituições públicas de ensino e pesquisa que participam dessas ações estão apresentadas na Tabela 2. Essa força-tarefa acontece em diversas instituições do país, contando com o apoio de voluntários capacitados e do governo para aumentar a capacidade de realização de testes de diagnósticos moleculares e sorológicos para o SARS-CoV-2.

\section{CONSIDERAÇÕES FINAIS}

$\mathrm{Na}$ busca de iniciativas que visam minimizar o impacto da pandemia causada pelo SARS-CoV-2, o diagnóstico ganha destaque por permitir que os pacientes infectados sejam corretamente identificados e que intervenções possam ser realizadas a fim de controlar a transmissão da doença. Com base nesta premissa, pesquisadores brasileiros somam esforços para encontrar soluções que auxiliem no diagnóstico da doença. 0 avanço das pesquisas nas universidades e em institutos de pesquisa brasileiros é de extrema importância e tem contribuído para o desenvolvimento de novos métodos que podem complementar os testes disponíveis atualmente. Não obstante, a colaboração entre instituições, universidades, governo e empresas privadas é necessária para que essas iniciativas possam ser consolidadas, de modo que as soluções desenvolvidas possam ser produzidas em escala e comercializadas atendendo aos anseios da saúde pública.

Vale ressaltar que além das iniciativas encontradas no período estipulado e que, portanto, foram divulgadas no presente trabalho, existe uma tendência de todas as universidades e institutos de pesquisa contribuírem no diagnóstico da COVID-19, como o caso da UFSJ. 

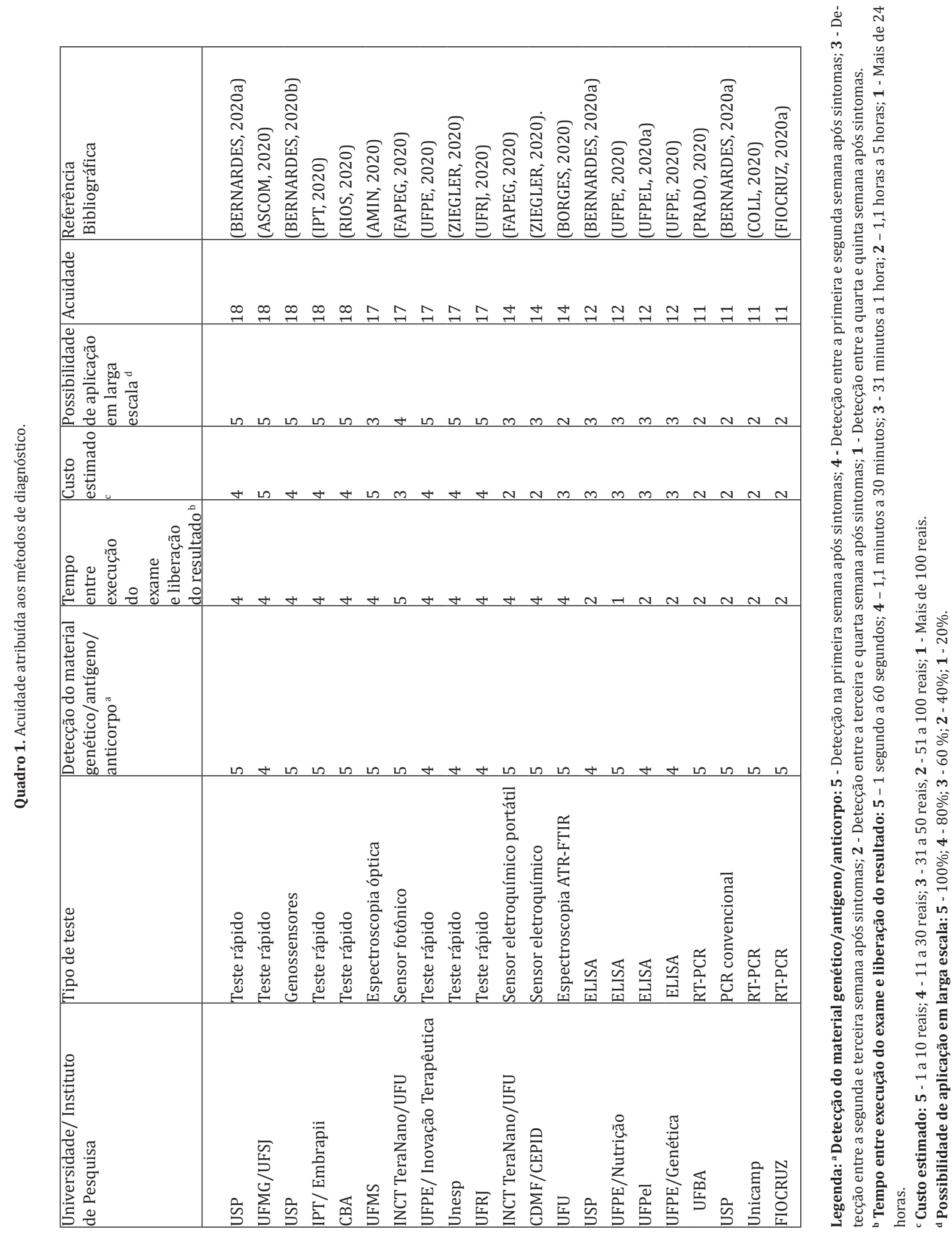
Tabela 2. Ações e estratégias das universidades e institutos de pesquisa para o combate da COVID-19.

\begin{tabular}{|c|c|}
\hline Instituição & Referência \\
\hline Universidade Federal de Minas Gerais & $\begin{array}{l}\text { (Espíndola, 2020b; NUPAD, 2020; UFMG, 2020a; UFMG, } \\
\text { 2020b) }\end{array}$ \\
\hline Universidade Federal de Viçosa & (UFV, 2020a; UFV, 2020b; UFV, 2020c) \\
\hline Universidade Federal dos Vales do Jequitinhonha e Mucuri & (UFVJM, 2020) \\
\hline Universidade Federal de Ouro Preto & (Souza, 2020) \\
\hline Fundação Oswaldo Cruz & (Castro, 2020; FIOCRUZ, 2020c; IBMP, 2020; Sobreira, 2020) \\
\hline Instituto Butantan & BUTANTAN, 2020 \\
\hline Universidade Federal do Rio Grande do Sul & (UFRGS, 2020a; UFRGS, 2020b) \\
\hline Universidade Federal da Paraíba & (UFPB, 2020) \\
\hline Universidade Federal do Ceará & (UFC,2020a; UFC,2020b) \\
\hline Universidade Federal do Pernambuco & (UFPE,2020) \\
\hline Universidade Federal do Rio Grande do Norte & (Rebouças, 2020; Silva, 2020) \\
\hline Universidade Federal de Ciências da Saúde de Porto Alegre & (UFCSPA, 2020a; UFCSPA, 2020b; UFCSPA, 2020c) \\
\hline Universidade Federal do Rio Grande & (64-ANDIFES, 2020) \\
\hline Universidade Federal de Santa Catarina & (UFSC, 2020). \\
\hline Universidade Federal de Mato Grosso do Sul & (Comineti, 2020; Oliveira, 2020) \\
\hline Universidade Federal de Santa Maria & (UFSM, 2020; Henriques, 2020) \\
\hline Universidade Federal de Juiz de Fora & (APESJF, 2020; UFJF,2020) \\
\hline Universidade Federal de Pelotas & (UFPEL, 2020b) \\
\hline Universidade Federal do Amazonas & (Siqueira, 2020) \\
\hline $\begin{array}{l}\text { Universidade Regional do Noroeste do Estado do } \\
\text { Rio Grande do Sul }\end{array}$ & (UNIJUÍ, 2020) \\
\hline Universidade Federal de Goiás & (UFG, 2020) \\
\hline Universidade Federal do Triângulo Mineiro & (UFTM, 2020) \\
\hline Instituto Federal de Goiás & $(\mathrm{IFG}, 2020)$ \\
\hline Universidade Federal do Paraná & (UFPR,2020) \\
\hline Universidade Federal de Mato Grosso & (UFMT, 2020) \\
\hline Universidade Federal de São Carlos & (Salmázio, 2020) \\
\hline Universidade Estadual Paulista & $\begin{array}{l}\text { (Marinho, 2020a, Marinho, 2020b; } \\
\text { Mazzitelli; Hoyo, 2020; UNESP, 2020) }\end{array}$ \\
\hline Universidade Estadual do Centro-Oeste & (UNICENTRO, 2020) \\
\hline Universidade do Estado do Amazonas & (UEA, 2020) \\
\hline Universidade Estadual de Londrina & (Livoratti, 2020a; Livoratti, 2020b) \\
\hline Universidade Estadual de Ponta Grossa & (UEPG, 2020) \\
\hline Universidade Municipal de São Caetano do Sul & (RIBEIRO, 2020) \\
\hline Universidade Federal do Pampa & (Da Silveira, 2020a, Da Silveira 2020b) \\
\hline Universidade Estadual de Montes Claros & (UNIMONTES, 2020) \\
\hline Universidade Federal do Espírito Santo & (Marinho, 2020c) \\
\hline Universidade Federal do Cariri & (UFCA, 2020) \\
\hline Universidade Federal do Pará & (Trajano, 2020) \\
\hline Universidade Federal do Piauí & (UFPI, 2020) \\
\hline Universidade Federal de Alagoas & (Gonzaga, 2020) \\
\hline Universidade de Brasília & (UNB, 2020) \\
\hline Universidade do Estado de Santa Catarina & (Da Silva, 2020) \\
\hline Universidade Federal da Integração Latino-Americana & (UNILA, 2020) \\
\hline
\end{tabular}




\section{REFERÊNCIAS}

ADHIKARI, S. P.; MENG, S.; WU, Y. et al. Epidemiology, causes, clinical manifestation and diagnosis, prevention and control of coronavirus disease (COVID-19) during the early outbreak period: a scoping review. Infect Dis Poverty., v. 1, n. 9, p. 1-12, 2020.

AMIN, V. Pesquisadores propõem método para diagnóstico rápido da Covid-19. UFMS, Mato Grosso do Sul, 2020. Disponível em: <https://www.ufms.br/pesquisadores-propoem-metodo-para-diagnostico-rapido-da-covid-19/>. Acesso em: $30 \mathrm{abr}$ 2020 .

ANDIFES. Associação Nacional dos Dirigentes das Instituições Federais de Ensino Superior. FURG e HU/Ebserh vão realizar quatro mil testes moleculares para diagnóstico do coronavírus em Rio Grande. Rio Grande, RS: Andifes; 2020. Disponível em: <http://www.andifes.org.br/furg-e-hu-ebserh-vao-realizar-quatro-mil-testes-moleculares-para-diagnostico-do-coronavirus-em-rio-grande/>. Acesso em: 30 abr. 2020.

APESJF. Associação dos Professores do Ensino Superior de Juiz de Fora. UFJF realiza testes de diagnósticos de COVID-19 - confira entrevista com prof. Lyderson Viccini (ICB). Juiz de Fora, MG: APESJF; 2020. Disponível em: <https://www.apesjf.org. $\mathrm{br} /$ ufj-realiza-testes-de-diagnosticos-de-covid-19-confira-entrevista-com-prof-lyderson-viccini-icb>. Acesso em: 1 mai. 2020 .

ARRUDAS, M. Unicamp e USP desenvolvem teste rápido e barato para identificar coronavírus. USP, São Paulo, 2020. Disponível em: <http://www.inovacao.usp.br/unicamp-e-usp-desenvolvem-teste-rapido-e-barato-para-identificar-coronavirus/>. Acesso em: 08 jun. 2020.

ASCOM. Pesquisa da UFSJ e UFMG busca desenvolver testes rápidos para Covid-19. UFSJ, São João Del-Rei, MG, 2020. Disponível em: <https://ufsj.edu.br/noticias_ler.php?codigo_noticia=7921 >. Acesso em: 01 mai. 2020.

BERNARDES, J. Biossensores que detectam câncer também poderão diagnosticar coronavírus. USP, São Paulo, 2020b. Disponível em: <https://jornal.usp.br/ciencias/biossensores-que-detectam-cancer-tambem-poderao-diagnosticar-coronavirus/>. Acesso em: 30 abr. 2020.

BERNARDES, J. Testes desenvolvidos na USP tornarão diagnóstico da covid-19 mais acessível. USP, São Paulo, 2020a. Disponível em: <https://jornal.usp.br/ciencias/testes-desenvolvidos-na-usp-tornarao-diagnostico-da-covid-19-mais-rapido-e-acessivel>. Acesso em: 19 abr. 2020.

BORGES, D. Cientistas da UFU e do Canadá propõem diagnóstico do coronavírus pela saliva. UFU, Uberlândia, 2020. Disponível em: <http://comunica.ufu.br/noticia/2020/02/cientistas-da-ufu-e-do-canada-propoem-diagnostico-do-coronavirus-pela-saliva>. Acesso em: 01 mai. 2020.
BUTANTAN. Instituto Butantan. Governo de SP recebe mais 575 mil testes para Covid-19 importados da Coreia do Sul. São Paulo, SP: instituto Butantan; 2020. Disponível em: <http://www. butantan.gov.br/noticias/governo-de-sp-recebe-mais-575-mil-testes-para-covid-19-importados-da-coreia-do-sul>. Acesso em: 7 mai. 2020.

CASTRO R. Fiocruz implanta Unidades de Apoio ao Diagnóstico da Covid-19. Belo Horizonte, MG: FIOCRUZ; 2020. Disponível em: <https://portal.fiocruz.br/noticia/fiocruz-implanta-unidades-de-apoio-ao-diagnostico-da-covid-19>. Acesso em: 7 mai. 2020.

COLL, L. Pesquisadores da Unicamp iniciam elaboração de teste para diagnóstico local do coronavírus. UNICAMP, Campinas, 2020. Disponível em: <https://www.unicamp.br/unicamp/ noticias/2020/03/17/pesquisadores-da-unicamp-iniciam-elaboracao-de-teste-para-diagnostico-local-do >. Acesso em: 01 mai.2020.

COMINETI, A. Laboratório será usado para diagnóstico e pesquisa da Covid-19 em Três Lagoas. Mato Grosso do Sul: UFMS; 2020. Disponível em: <https://www.ufms.br/laboratorio-sera-usado-para-diagnostico-e-pesquisa-da-covid-19-em-tres-lagoas/>. Acesso em: 30 abr. 2020.

CUBAS, J. Pesquisadores da UFPR e da UFOP desenvolvem diagnóstico de Covid-19 por imagens de Raio-X. UFPR, Paraná, 2020. Disponível em: <https://www.ufpr.br/portalufpr/noticias/pesquisadores-da-ufpr-e-da-ufop-desenvolvem-diagnostico-de-covid-19-por-imagens-de-raio-x/>. Acesso em: 08 jun. 2020 .

CUI, J.; LI, F.; SHI, Z. L. Origin and evolution of pathogenic coronaviruses. Nat Rev Microbiol., v. 17, n. 3, p. 181-192, 2019.

DALLANHOL, H. Aluno da UFSC pesquisa modelo de detector da Covid-19 por meio de radiografias e tomografias. UFSC, Florianópolis, 2020. Disponível em: <https://noticias.ufsc. br/2020/03/aluno-da-ufsc-pesquisa-modelo-de-detector-da-covid-19-por-meio-de-radiografias/>. Acesso em: 08 jun. 2020.

DA SILVA, T. Udesc Lages cede equipamento para Lacen para realização de testes de Covid-19. Florianópolis, SC: Udesc; 2020. Disponível em: <https://www.udesc.br/cav/noticia/ udesc_lages_cede_equipamento_para_lacen_para_realizacao_ de_testes_de_covid-19>. Acesso em: 4 jun. 2020.

DA SILVEIRA, A. Começam testes rápidos para estudo sobre o perfil da população infectada pelo coronavírus. Bagé, RS: Unipampa; 2020a. Disponível em: <https://unipampa.edu.br/ portal/comecam-testes-rapidos-para-estudo-sobre-o-perfil-da-populacao-infectada-pelo-coronavirus >. Acesso em: 7 jun. 2020.

DA SILVEIRA, A. Testes rápidos para coronavírus serão aplicados em Bagé nesta sexta-feira, 8. Bagé, RS: Unipampa; 2020b. 
Disponível em: <https://unipampa.edu.br/portal/testes-rapidos-para-coronavirus-serao-aplicados-em-bage-nesta-sexta-feira-8>. Acesso em: 7 jun. 2020.

ESPINDOLA M. Laboratórios da UFMG poderão auxiliar nos testes de diagnóstico da Covid-19. Belo Horizonte, MG: UFMG; 2020b. Disponível em: <https://ufmg.br/comunicacao/noticias/laboratorios-da-ufmg-poderao-auxiliar-nos-testes-para-deteccao-da-infeccao-pelo-coronavirus $>$. Acesso em: 1 mai. 2020.

ESPINDOLA, M. Software que identifica padrões viróticos é desenvolvido na UFMG. UFMG, Belo Horizonte, 2020a. Disponível em: <https://ufmg.br/comunicacao/noticias/software-que-identifica-padroes-viroticos-e-desenvolvido-na-ufmg $>$. Acesso em: 07 mai. 2020.

FAPEG. Fundação de Amparo à Pesquisa do Estado de Goiás. INCT desenvolve novos sensores para diagnóstico da Covid-19. Goiânia, FAPEG, Goiás, 2020. Disponível em: <http://www.fapeg.go.gov.br/inct-desenvolve-novos-sensores-para-diagnostico-da-covid-19/>. Acesso em: 30 abr. 2020

FIOCRUZ.Fundação Oswaldo Cruz. Fiocruz produzirá kits para diagnóstico do novo coronavírus. FIOCRUZ, Belo Horizonte, 2020a. Disponível em: <https://portal.fiocruz.br/noticia/fiocruz-produzira-kits-para-diagnostico-do-novo-coronavirus>. Acesso em: 03 mai. 2020

FIOCRUZ. Fundação Oswaldo Cruz. Fiocruz Minas vai atuar no diagnóstico de Covid-19. Belo Horizonte, MG: FIOCRUZ; 2020b. Disponível em: <https://portal.fiocruz.br/noticia/fiocruz-minas-vai-atuar-no-diagnostico-de-covid-19>. Acesso em: 3 mai. 2020.

GOMES, R. UnB desenvolve sistema para análise de raio-x com inteligência artificial. UnB, Brasília, 2020. Disponível em: <https://noticias.unb.br/117-pesquisa/4059-unb-desenvolve-sistema-para-analise-de-raio-x-com-inteligencia-artificial>. Acesso em: 01 mai. 2020.

GONZAGA, T. Pesquisadores da Ufal vão ajudar na realização de testes de covid-19. Maceió, AL: Ufal; 2020. Disponível em: <https://ufal.br/ufal/noticias/2020/4/pesquisadores-da-ufal-vao-ajudar-na-realizacao-de-testes-de-covid-19>. Acesso em: 1 mai. 2020.

GUO, L.; REN, L.; YANG, S. et al. Profiling Early Humoral Response to Diagnose Novel Coronavirus Disease (COVID-19). Clin Infec Dis., 2020.

HENRIQUES, M. Testes para Covid-19 começam a ser feitos na UFSM. Santa Maria, RS: UFSM; 2020. Disponível em: <https:// www.ufsm.br/unidades-universitarias/ce/2020/05/04/testes-para-covid-19-comecam-a-ser-feitos-na-ufsm/>. Acesso em: 20 abr. 2020
IBMP. Instituto de Biologia Molecular do Paraná. Fiocruz, em parceria com IBMP e Tecpar, implanta Unidade de Apoio ao Diagnóstico Covid-19 em Curitiba. Curitiba, PR: IBMP; 2020 Disponível em: <http://www.ibmp.org.br/pt-br/fiocruz-em-parceria-com-ibmp-e-tecpar-implanta-unidade-de-apoio-ao-diagnostico-covid-19-em-curitiba/>. Acesso em: 7 mai. 2020

IFG. Instituto Federal de Goiás. Servidores do Câmpus Goiânia do IFG participam de ação junto à UFG para realização de diagnósticos da Covid-19. Goiânia, GO: IFG; 2020. Disponível em: <https://www.ifg.edu.br/ultimas-noticias-campus-goiania/17854-servidores-do-campus-goiania-do-ifg-participam-de-acao-junto-a-ufg-para-a-realizacao-de-diagnosticos-da-covid-19>. Acesso em: 1 mai. 2020

INPI, Instituto Nacional da Propriedade Industrial. Trâmite prioritário. Rio de Janeiro, jun 2020. Disponível em: <https:// www.gov.br/inpi/pt-br/servicos/patentes/tramite-prioritario/acelere-seu-exame>. Acesso em: 01 jun. 2020.

IPT. Instituto de Pesquisas Tecnológicas. Teste rápido identifica Covid-19 com segurança. IPT, São Paulo, 2020. Disponível em: <https://www.ipt.br/noticia/1620_teste_rapido_identifica_ covid_19_com_seguranca.htm>. Acesso em: 01 mai. 2020.

LEE, C. Y.; LIN, R. T. P.; RENIA, L. et al. Serological Approaches for COVID-19: Epidemiologic Perspective on Surveillance and Control. Front. Immunol v.11, p.879, 2020.

LIVORATTI, P. Hospital Universitário da UEL realiza testes Padrão Ouro para COVID 19 e envia amostras para validação pelo Lacen. Londrina, PA: UEL; 2020b. Disponível em: <http:// www.uel.br/com/agenciaueldenoticias/index.php?arq=ARQ not\&id=30309>. Acesso em: 4 jun. 2020.

LIVORATTI, P. Laboratório de Análises Clínicas do HU/UEL faz testes rápidos para detecção de anticorpos de coronavírus. Londrina, PA: UEL; 2020a. Disponível em:< http://www.uel. $\mathrm{br} / \mathrm{com} /$ agenciaueld enoticias/index.php?arq=ARQ not\&id=30231>. Acesso em: 4 jun. 2020.

LONG, Q. X.; LIU, B. Z.; DENG, H. J. et. al. Antibody responses to SARS-CoV-2 in patients with COVID-19. Nat Med. v.26, p. 845848,2020

MARINHO, J. Unesp constitui centros de referência em testes de Covid-19. São Paulo, SP: UNESP; 2020a. Disponível em: <https://www2.unesp.br/portal\#!/noticia/35635/unesp-constitui-centros-de-referencia-em-testes-de-covid-19/>. Acesso em: 4 jun. 2020

MARINHO, J. Unesp se une ao Adolfo Lutz em Rio Claro para ampliar testagem. São Paulo, SP: UNESP; 2020b. Disponível em: <https://www2.unesp.br/portal\#!/noticia/35716/unesp-se-une-ao-adolfo-lutz-em-rio-claro-para-ampliar-testagem/> Acesso em: 4 jun. 2020 
MARINHO, T. Ufes cede equipamentos para diagnóstico da COVID-19 ao Laboratório Central da Sesa. Vitória, ES: UFES 2020c. Disponível em:<http://coronavirus.ufes.br/conteudo/ ufes-cede-equipamentos-para-diagnostico-da-covid-19-ao-laboratorio-central-da-sesa>. Acesso em: 30 abr. 2020 .

MAZZITELLI, F, HOYO, N. DEL. Parceria Unesp-Embraer viabiliza 40 mil testes no interior de SP. São Paulo, SP: UNESP; 2020. Disponível em: <https://www2.unesp.br/portal\#!/noticia/35742/parceria-unesp-embraer-viabiliza-40-mil-testes-no-interior-de-sp/>. Acesso em: 4 jun. 2020.

NUPAD. Núcleo de Ações e Pesquisa em Apoio diagnóstico da Faculdade de Medicina da UFMG. Nupad é mais um laboratório da UFMS a auxiliar no diagnóstico de Covid-19 em Minas Gerais. NUPAD, Belo Horizonte, 2020. Disponível em:< https:// www.nupad.medicina.ufmg.br/nupad-e-mais-um-laboratorio-da-ufmg-a-auxiliar-no-diagnostico-de-covid-19-em-minas-gerais/ > Acesso em: 1 mai. 2020.

OLIVEIRA, T. UFMS analisa mais de 500 amostras do Drive-Thru Covid-19. Campo Grande, MS: UFMS; 2020. Disponível em: <https://www.ufms.br/ufms-analisa-mais-de-500-amostras-do-drive-thru-covid-19/>. Acesso em: 1 mai. 2020.

PRADO, L. Exame rápido para coronavírus da UFBA: como funciona? UFBA, Salvador, BA, 2020. Disponível em: <https:// www.sanarmed.com/ufba-e-o-exame-para-coronavirus-como-funciona-colunistas>. Acesso em: 01 mai. 2020.

PUPIM, P. Cientistas criam método para identificar a Covid-19 com imagens de raio-x. UEM, Maringá, 2020. Disponível em: $<$ http://www.asc.uem.br/index.php?option=com_content\&vi ew $=$ article\&id $=24464$ : cientistas-da-uem-criam-metodo-para-identificar-a-covid-19-com-imagens-de-raio-x\&catid=986\&Itemid=210 >. Acesso em: 01 mai. 2020.

REBOUÇAS, J. DE P. UFRN adquire kits e começa a testagem para Covid-19. Natal, RN: UFRN; 2020. Disponível em: <https://ufrn.br/imprensa/noticias/34510/ufrn-adquire-kits-e-comeca-a-testagem-para-covid-19>. Acesso em: 30 abr. 2020.

RIBEIRO, M. Prefeitura, USCS e USP iniciam programa de testagem domicial contra o coronavírus. São Caetano do Sul, SP; 2020. Disponível em: <http://www.saocaetanodosul.sp.gov. $\mathrm{br} /$ noticias-scs/prefeitura-uscs-e-usp-iniciam-programa-de-testagem-domiciliar-contra-o-coronavirus.html>. Acesso em: 7 jun. 2020.

RIOS, L. CBA trabalha na produção de testes rápidos da Covid-19 com insumos nacionais. SUFRAMA, Manaus, 2020. Disponível em: <http://site.suframa.gov.br/noticias/cba-trabalha-na-producao-de-testes-rapidos-da-covid-19-com-insumos-nacionais>. Acesso em: 19 abr. 2020.

RODRIGUEZ-MORALES, A. J; CARDONA, O. J. A.; GUTIÉRREZ E. O. et al. Clinical, laboratory and imaging features of COVID-19: A systematic review and meta-analysis. Travel Med Infect Dis., v. 34, p.101623, 2020.
ROTHAN, H. A.; BYRAREDDY, S. N. The epidemiology and pathogenesis of coronavirus disease (COVID-19) outbreak. J Autoimmun., v. 109, n. 102433, 2020.

SALMÁZIO, F. UFSCar realiza primeiros exames para COVID-19. São Carlos, SP: UFSCAR; 2020. Disponível em: <https://www2. ufscar.br/noticia?codigo=12637>. Acesso em: 4 jun. 2020.

SANTOS, M. UFOP desenvolve projeto para melhorar detecção da Covid-19. UFOP, Ouro Preto, 2020. Disponível em: <https:// ufop.br/noticias/pesquisa-e-inovacao/ufop-desenvolve-projeto-para-melhorar-deteccao-da-covid-19>. Acesso em: 01 mai. 2020.

SILVA, W. UFRN realiza quase 2 mil testes da Covid-19. Natal, RN: UFRN; 2020. Disponível em: <https://www.ufrn.br/imprensa/noticias/35011/ufrn-realiza-quase-2-mil-testes-da-covid-19>. Acesso em: 30 abr. 2020.

SIQUEIRA, S. Ufam tem centro de testes rápidos para profissionais de saúde. Manaus, AM: UFAM; 2020. Disponível em: <https://www.ufam.edu.br/noticias-coronavirus/1306-ufam-tera-centro-de-testes-rapidos-para-profissionais-de-saude. html>. Acesso em: 1 mai. 2020.

SOBREIRA, A. Fiocruz Ceará cede equipamentos para ampliar capacidade de testes de Covid-19. Eusébio, CE: FIOCRUZ; 2020. Disponível em: <https://portal.fiocruz.br/noticia/fiocruz-ceara-cede-equipamentos-para-ampliar-capacidade-de-testes-de-covid-19>. Acesso em: 30 abr. 2020.

SOUZA L. UFOP assina termo de compromisso para fazer parte da RedeLab Covid-19. Ouro Preto, MG: UFOP; 2020. Disponível em: <https://ufop.br/noticias/coronavirus/ufop-assina-termo-de-compromisso-para-fazer-parte-da-redelab-covid-19>. Acesso em: 4 jun. 2020.

TORTORICI, M. A.; Veesler, D. Structural insights into coronavirus entry. Adv Virus Res., v. 105, p. 93-116, 2019.

TRAJANO, M. UFPA colabora com a realização de testes para o diagnóstico do coronavírus. Belém, PA: UFPA; 2020. Disponível em: <https://portal.ufpa.br/index.php/ultimas-noticias2/11465-ufpa-colabora-na-realizacao-de-testes-para-o-diagnostico-do-coronavirus>. Acesso em: 1 mai. 2020.

UDUGAMA, B.; KADHIRESAN, P.; KOZLOWSKI, H. N. et al. Diagnosing COVID-19: The Disease and Tools for Detection. ACS Nano., v. 14, n. 4, p. 3822-3835, 2020.

UEA. Universidade do Estado do Amazonas. Em parceria com a FVS, UEA realiza teste rápido em Drive Thru para profissionais de saúde. Manaus, AM: UEA; 2020. Disponível em: <https://covid19.uea.edu.br/em-parceria-com-a-fvs-uea-realiza-teste-rapido-em-drive-thru-para-profissionais-de-saude/>. Acesso em: 4 jun. 2020.

UEPG. Universidade Estadual de Ponta Grossa. UEPG adquire testes de Covid-19 para Hospital Universitário. Ponta Grossa, 
PR: UEPG; 2020. Disponível em: < .br/testes-covid-19-hospital-universitario/>. Acesso em: 7 jun. 2020.

UERJ. Universidade do Estado do Rio de Janeiro. Pesquisa de professores da Uerj facilita diagnóstico e acompanhamento de pacientes com Covid-19. UERJ, Rio de Janeiro, 2020. Disponível em: <https://www.uerj.br/noticia/10912/>. Acesso em: 01 mai. 2020 .

UFC. Universidade Federal do Ceará. UFC é certificada pelo LACEN e inicia testes de COVID-19; expectativa é de pelo menos 300 testes por dia. Fortaleza, CE: UFC; 2020a. Disponível em: <http://www.ufc.br/noticias/14464-ufc-e-certificada-pelo-lacen-e-inicia-testes-de-covid-19-expectativa-e-de-pelo-menos-300-testes-por-dia>. Acesso em: 4 jun. 2020.

UFC. Universidade Federal do Ceará. UFC disponibiliza equipamento e pesquisadores para diagnóstico de COVID-19. Fortaleza, CE: UFC; 2020b. Disponível em: <http://www.ufc.br/ noticias/14423-ufc-disponibiliza-equipamento-e-pesquisadores-para-diagnostico-de-covid-19>. Acesso em: 1 mai. 2020.

UFCA. Universidade Federal do Cariri. UFCA cede laboratório e equipamentos para acelerar diagnósticos de Covid-19 no Cariri. Juazeiro do Norte, CE: UFCA; 2020. Disponível em: <https:// www.ufca.edu.br/noticias/ufca-cede-laboratorio-e-equipamentos-para-acelerar-diagnosticos-de-covid-19-no-cariri/>. Acesso em: 30 abr. 2020.

UFCSPA. Universidade Federal de Ciências da Saúde de Porto Alegre. UFCSPA e Santa Casa atuam no diagnóstico de Covid-19. Porto Alegre, RS: UFCSPA; 2020a. Disponível em: <https:// www.ufcspa.edu.br/index.php/coronavirus/8280-ufcspa-e-santa-casa-atuam-no-diagnostico-de-covid-19>. Acesso em: 7 jun. 2020.

UFCSPA. Universidade Federal de Ciências da Saúde de Porto Alegre. Nas ruas para testar a população. Porto Alegre, RS: UFCSPA; 2020b. Disponível em: <https://www.ufcspa.edu.br/ index.php/coronavirus/8410-nas-ruas-para-testar-a-populacao>. Acesso em: 7 jun. 2020.

UFCSPA. Universidade Federal de Ciências da Saúde de Porto Alegre. UFCSPA integra estudo de Covid-19 com a população de Esteio. Porto Alegre, RS: UFCSPA; 2020c. Disponível em: <https://www.ufcspa.edu.br/index.php/ultimas-noticias/34-noticias/8351-ufcspa-integra-estudo-de-covid-19-com-a-populacao-de-esteio>. Acesso em: 7 jun. 2020.

UFG. Universidade Federal de Goiás. Coronavírus: a UFG está de olho. Previna-se! Goiânia, GO: UFG; 2020. Disponível em: <https://https://ufg.br/n/124654-coronavirus-a-ufg- esta-de-olho-previna-se>. Acesso em: 1 mai. 2020.

UFJF. Universidade Federal de Juiz de Fora. UFJF inicia realização de testes para diagnóstico de Covid-19. Juiz de Fora, MG: UFJF; 2020. Disponível em: <https://www2.ufjf.br/noticias/2020/04/16/ufjf-inicia-realizacao-de-testes-para-diagnostico-de-covid-19>. Acesso em: 1 mai. 2020
UFMG. Universidade Federal de Minas Gerais. Laboratórios da UFMG começam a fazer testes de diagnóstico. Belo Horizonte, MG: UFMG; 2020a. Disponível em: <https://www.medicina. ufmg.br/laboratorios-da-ufmg-comecam-a-fazer-testes-de-diagnostico-do-novo-coronavirus>. Acesso em: 1 mai. 2020.

UFMG. Universidade Federal de Minas Gerais. UFMG amplia capacidade de realização de testes para detectar o coronavírus Belo Horizonte, MG: UFMG; 2020b. Disponível em: <https:// ufmg.br/comunicacao/noticias/ufmg-amplia-sua-capacidade-de-realizacao-de-testes>. Acesso em: 1 mai. 2020.

UFMT. Universidade Federal de Mato Grosso. HUJM, Favet e FM articulam força-tarefa para disponibilizar testes de Covid-19 à comunidade hospitalar. Cuiabá, MT: UFMT; 2020. Disponível em: <https://www.ufmt.br/ufmt/site/noticia/visualizar/47975/Cuiaba>. Acesso em: 1 mai. 2020.

UFPB. Universidade Federal da Paraíba. Governo e UFPB firmam parceria para ampliar diagnóstico da Covid-19. João Pessoa, PB: UFPB; 2020. Disponível em: <https://paraiba.pb.gov. $\mathrm{br} /$ noticias/governo-e-ufpb-firmam-parceria-para-ampliar-diagnostico-da-covid-19>. Acesso em: 1 mai. 2020.

UFPE. Universidade Federal de Pernambuco. Diagnóstico e Identificação do Vírus. UFPE, Recife, 2020. Disponível em: <https://www.ufpe.br/covid-19/observatorio/diagnostico>. Acesso em: 1 mai. 2020

UFPEL.Universidade Federal de Pelotas. UFPel desenvolve pesquisa para diagnóstico sorológico da COVID-19. UFPel, Pelotas, 2020. Disponível em: <https://ccs2.ufpel.edu.br/ wp/2020/04/01/ufpel-desenvolve-pesquisa-para-diagnostico-sorologico-da-covid-19>. Acesso em: 1 mai. 2020.

UFPEL. Universidade Federal de Pelotas. UFPel fará testes de RT-PCR para COVID-19. Pelotas, RS: UFPEL; 2020. Disponível em: <https://ccs2.ufpel.edu.br/wp/2020/04/05/ufpel-fara-testes-de-rt-pcr-para-covid-19>. Acesso em: 1 mai. 2020.

UFPI. Universidade Federal do Piauí. A UFPI cede equipamentos para auxiliar de forma mais rápida os testes do COVID - 19 Teresina, PI: UFPI; 2020. Disponível em:< https://ufpi.br/noticias-coronavirus/35838-a-ufpi-cede-equipamentos-para-auxiliar-de-forma-mais-rapida-os-teste-do-covid-19>. Acesso em: 30 abr 2020 .

UFPR. Universidade Federal do Paraná. Complexo HC é habilitada para diagnóstico de Covid-19. Curitiba, PR: UFPR; 2020 Disponível em: <http://www2.ebserh.gov.br/web/chc-ufpr/ noticias/-/asset_publisher/kolvfeKgK2VF/content/ id/5099913/2020-04-omplexo-hc-avanca-no-diagnostico-para-covid-19>. Acesso em: 1 mai. 2020.

UFRGS. Universidade Federal do Rio Grande do Sul. UFRGS vai realizar até 500 testes de Covid-19 por dia. Porto Alegre, RS UFRGS; 2020a. Disponível em: <http://www.ufrgs.br/ufrgs/ noticias/ufrgs-vai-realizar-ate-500-testes-de-covid-19-por-dia>. Acesso em: 30 abr. 2020. 
UFRGS. Universidade Federal do Rio Grande do Sul. Voluntários começam a realizar os testes de diagnóstico da Covid-19 na UFRGS - Coronavírus. Porto Alegre, RS: UFRGS; 2020b. Disponível em: <https://www.ufrgs.br/coronavirus/base/voluntarios-comecam-a-realizar-os-testes-de-diagnostico-da-covid-19-na-ufrgs>. Acesso em: 30 abr. 2020.

UFRJ. Universidade Federal do Rio de Janeiro desenvolve novo teste para detectar COVID-19. UFRJ, Rio de Janeiro, 2020. Disponível em: <https://ufrj.br/noticia/2020/03/23/coronavirus-ufrj-desenvolve-novo-teste-para-detectar-covid-19>. Acesso em: 19 abr. 2020.

UFRRJ. Universidade Federal Rural do Rio de Janeiro. Pesquisadores da UFRRJ criam aplicativo para auxiliar o diagnóstico da Covid-19. UFRRJ, Seropédica, 2020. Disponível em: <http:// coronavirus.ufrrj.br/pesquisadores-da-ufrrj-criam-aplicativo-para-auxiliar-o-diagnostico-da-covid-19>. Acesso em: 04 jun. 2020.

UFSC. Universidade Federal de Santa Catarina. Equipe da força-tarefa da UFSC informa sobre a realização de exames para Covid-19. Florianópolis, SC: UFSC; 2020. Disponível em: <https:// lbmms.paginas.ufsc.br/2020/04/06/equipe-da-forca-tarefa-da-ufsc-informa-sobre-a-realizacao-de-exames-para-covid-19>. Acesso em: 30 abr. 2020.

UFSM. Universidade Federal de Santa Maria. Campus da UFSM em Palmeira das Missões inicia testes para Covid-19. Santa Maria, RS: UFSM; 2020. Disponível em: <https://www.ufsm. br/2020/04/28/campus-da-ufsm-em-palmeira-das-missoes-inicia-testes-para-covid-19>. Acesso em: 30 abr. 2020.

UFTM. Universidade Federal do Triângulo Mineiro. UFTM, Mosaic e FUNEPU firmam parceria para disponibilizar 10 mil testes que detectam o novo coronavírus. Uberaba, MG: UFTM; 2020. Disponível em: <http://www.uftm.edu.br/ultimas-noticias/2442-uftm-mosaic-e-funepu-firmam-parceria-para-disponibilizar-10-mil-testes-que-detectam-o-novo-coronavirus>. Acesso em: 4 jun. 2020.

UFV. Universidade Federal de Viçosa. UFV poderá realizar testes para detecção do coronavírus. Viçosa, MG: UFV; 2020a. Disponível em: <https://www2.dti.ufv.br/noticias/scripts/exibeNoticiaMulti.php?codNot=32304\&link=corpo $>$. Acesso em: 1 mai. 2020

UFV. Universidade Federal de Viçosa. UFV começa a realizar primeiros testes para detecção do coronavírus. Viçosa, MG: UFV; 2020b. Disponível em: <https://www2.dti.ufv.br/noticias/scripts/exibeNoticiaMulti.php?codNot=32392>. Acesso em: 1 mai. 2020.

UFV. Universidade Federal de Viçosa. Laboratórios da UFV recebem certificado da Funed pela qualidade dos testes de detecção do novo coronavírus. Viçosa, MG: UFV; 2020c. Disponível em: <https://www2.dti.ufv.br/noticias/scripts/exibeNoticiaMulti.php?codNot=32419>. Acesso em: 1 mai. 2020.
UFVJM. Universidade Federal dos Vales do Jequitinhonha e Mucuri. Coronavírus - UFVJM pronta para realizar testes e ajudar no combate à doença. Diamantina, MG: UFVJM; 2020. Disponível em: <http://portal.ufvjm.edu.br/noticias/2020/coronavirus-ufvjm-pronta-para-realizar-testes-e-ajudar-no-combate-a-doenca>. Acesso em: 1 mai. 2020.

UNB. Universidade de Brasília. UnB ajuda a ampliar diagnósticos e rede de atendimento a casos da Covid-19. Brasília, DF: UnB; 2020. Disponível em:< https://www.noticias.unb. br/76-institucional/4058-unb-ajuda-a-ampliar-diagnosticos-e-rede-de-atendimento-a-casos-da-covid-19>. Acesso em: 1 mai. 2020.

UNESP. Universidade Estadual Paulista. Laboratório de Botucatu é credenciado pelo governo estadual. São Paulo, SP: Unesp; 2020. Disponível em: <https://www2.unesp.br/portal\#!/noticia/35659/laboratorio-de-botucatu-e-credenciado-pelo-governo-estadual>. Acesso em: 4 jun. 2020.

UNICENTRO. Universidade Estadual do Centro Oeste. Unicentro fará exames de detecção covid-19. Guarapuava, PR: Unicentro; 2020. Disponível em: <https://www3.unicentro.br/noticias/2020/04/01/unicentro-fara-exames-de-deteccao-covid-19>. Acesso em: 7 jun. 2020.

UNIJUÍ. Universidade Regional do Noroeste do Estado do Rio Grande do Sul. Unijuí realiza testes para a Covid-19. Ijuí, RS: Unijuí; 2020. Disponível em: <http://www.crub.org.br/blog/ unijui-realiza-testes-para-a-covid-19>. Acesso em: 1 mai. 2020.

UNILA. Universidade Federal da Integração Latino-Americana. UNILA e Prefeitura farão exames para detectar Covid-19, no Hospital Municipal. Foz do Iguaçu, PR: UNILA; 2020. Disponível em: < https://portal.unila.edu.br/noticias/unila-e-prefeitura-farao-exames-para-detectar-covid-19-no-hospital-municipal >. Acesso em: 1 mai. 2020.

UNIMONTES. Universidade Estadual de Montes Claros. Laboratório de Pesquisa em Saúde do HUCF/Unimontes credenciado para a realização de testes do Novo Coronavírus. Montes Claros, MG: Unimontes; 2020. Disponível em: < https://unimontes.br/laboratorio-de-pesquisa-em-saude-do-hucf-unimontes-credenciado-para-a-realizacao-de-testes-do-novo-coronavirus >. Acesso em: 4 jun. 2020.

UNIOESTE. Universidade Estadual do Oeste do Paraná. Huop inicia estudo com inteligência artificial para detectar Covid-19. Unioeste, Cascavel, 2020. Disponível em: <https://www.unioeste.br/portal/central-de-noticias/52709-huop-inicia-estudo-com-inteligencia-artificial-para-detectar-covid-19>. Acesso em: 1 mai. 2020.

USP. Universidade de São Paulo. Banco de imagens radiográficas contribui com diagnóstico da covid-19. USP, São Paulo, 2020. Disponível em: <https://jornal.usp.br/ciencias/banco-de-imagens-radiograficas-contribui-com-diagnostico-da-covid-19>. Acesso em: 7 mai. 2020. 
WEID, I. V. D. Panorama das patentes depositadas no INPI descrevendo métodos de diagnóstico para Coronavirus e outras viroses respiratórias. INPI, Rio de Janeiro, 2020. Disponível em: $<$ https://www.gov.br/inpi/pt-br/central-de-conteudo/ultimas-noticias/copy_of_observatorio-covid-19-faz-estudo-sobre-patentes-de-metodosdiagnosticos/patentes_diagnostico coronavirus_outrasviroses.pdf>. Acesso em: em 1 jun. 2020.

ZAINOL, R. Z.; OTHMAN, S.N.; ABDUL SAMAT, M.N. et al. Diagnostic performance of COVID-19 serology assays. Malays J Pathol. v.42, n.1, p.13-21, 2020.
ZIEGLER, M. F. Pesquisadores desenvolvem testes rápidos para detecção de COVID-19. FAPESP, São Paulo, 2020. Disponível em: <http://agencia.fapesp.br/pesquisadores-desenvolvem-testes-rapidos-para-deteccao-de-covid-19/32975/>. Acesso em: 6 mai. 2020

ZUCOLOTO, G.; MIRANDA, P.; PORTO, P. A propriedade industrial pode limitar o combate à pandemia? Ipea, Brasília, jun 2020. Disponível em: <https://www.ipea.gov.br/cts/pt/central-de-conteudo/artigos/artigos/188-a-propriedade-industrial-pode-limitar-o-combate-a-pandemia\#_ftn54>. Acesso em: 20 mai. 2020 